\title{
On the tautomerisation of porphycene on copper (111): Finding the subtle balance between van der Waals interactions and hybridisation
}

Dino Novko, Jean Christophe Tremblay, and María Blanco-Rey

Citation: The Journal of Chemical Physics 145, 244701 (2016); doi: 10.1063/1.4972213

View online: http://dx.doi.org/10.1063/1.4972213

View Table of Contents: http://aip.scitation.org/toc/jcp/145/24

Published by the American Institute of Physics

\section{Articles you may be interested in}

Self-consistent implementation of ensemble density functional theory method for multiple strongly correlated electron pairs

The Journal of Chemical Physics 145, 244104244104 (2016); 10.1063/1.4972174

Effective scheme for partitioning covalent bonds in density-functional embedding theory: From molecules to extended covalent systems

The Journal of Chemical Physics 145, 244103244103 (2016); 10.1063/1.4972012

An efficient quantum mechanical method for radical pair recombination reactions

The Journal of Chemical Physics 145, 244101244101 (2016); 10.1063/1.4972277

Si clusters are more metallic than bulk $\mathrm{Si}$

The Journal of Chemical Physics 145, 244302244302 (2016); 10.1063/1.4972813

Cu-metalated carbyne acting as a promising molecular wire

The Journal of Chemical Physics 145, 244702244702 (2016); 10.1063/1.4972867

Hyperfine interactions and internal rotation in methanol

The Journal of Chemical Physics 145, 244301244301 (2016); 10.1063/1.4972004

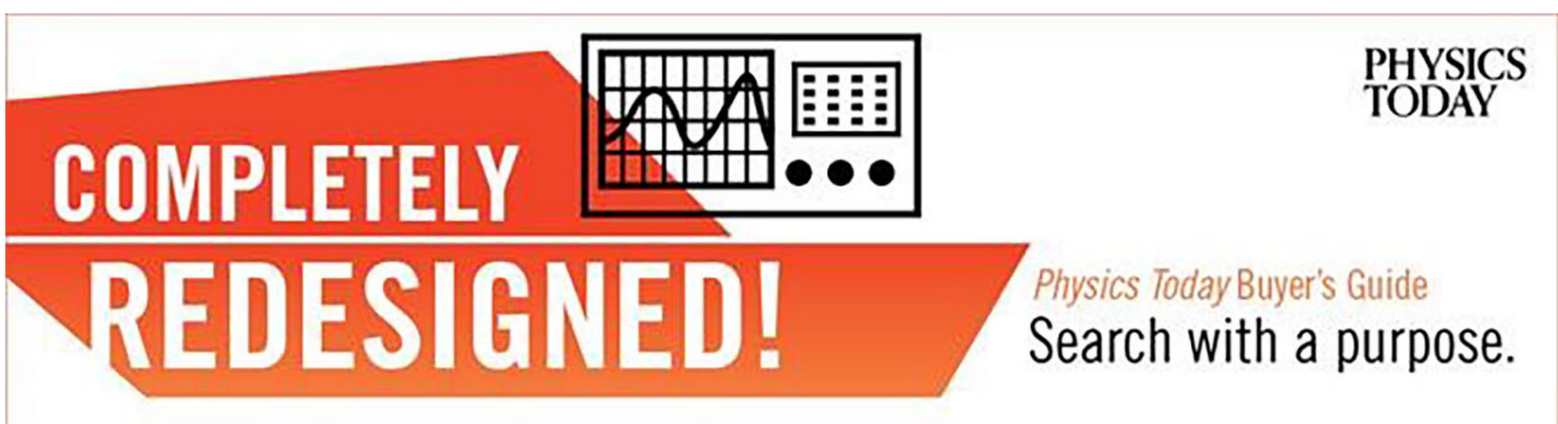




\title{
On the tautomerisation of porphycene on copper (111): Finding the subtle balance between van der Waals interactions and hybridisation
}

\author{
Dino Novko, ${ }^{1}$ Jean Christophe Tremblay, ${ }^{2, a)}$ and María Blanco-Rey ${ }^{1,3, b)}$ \\ ${ }^{1}$ Donostia International Physics Center (DIPC), Paseo Manuel de Lardizabal 4, \\ 20018 Donostia-San Sebastián, Spain \\ ${ }^{2}$ Institute for Chemistry and Biochemistry, Freie Universität Berlin, Takustraße 3, D-14195 Berlin, Germany \\ ${ }^{3}$ Departamento de Física de Materiales, Facultad de Químicas UPV/EHU, Apartado 1072, \\ 20018 Donostia-San Sebastián, Spain
}

(Received 27 September 2016; accepted 1 December 2016; published online 22 December 2016)

\begin{abstract}
We use density-functional theory (DFT) to analyse the interaction of trans- and cis-porphycene with $\mathrm{Cu}(111)$ and their interconversion by intramolecular $\mathrm{H}$-transfer. This tautomerisation reaction is characterised by small values for the reaction energy and barrier, on the order of $\sim 0.1 \mathrm{eV}$, where the trans configuration is thermodynamically more stable upon adsorption according to the experiments [J. N. Ladenthin et al., ACS Nano 9, 7287-7295 (2015)]. To gain even a qualitatively correct description of this reaction at the DFT level, an accurate treatment of dispersion interactions and a careful choice of the exchange contribution are required in order to predict the subtle energetics. Analysis of the electronic structure shows that adsorption is contributed by a van der Waals (vdW) interaction, mainly responsible for stabilising the polyaromatic fragments, and by a significant charge redistribution localised between $\mathrm{Cu}$ and the unsaturated $\mathrm{N}$ atoms of the molecule central cavity. We find that different $\mathrm{vdW}$ functionals can produce qualitatively different electronic structures, while yielding small trans vs. cis energy differences. Unlike other functionals surveyed here, vdW-DF with PBE exchange satisfactorily reproduces not only the experimental energetics but also the scanning tunneling microscopy images. This gives us confidence that this functional achieves a reliable balance between the two mechanisms contributing to the adsorption of porphycene. Published by AIP Publishing. [http://dx.doi.org/10.1063/1.4972213]
\end{abstract}

\section{INTRODUCTION}

A tautomerisation reaction is defined as an intramolecular transfer of hydrogen between two sites of an organic molecule, ${ }^{1}$ and the isomers convertible by this reaction type are known as tautomers. Tautomers, unlike enantiomers, behave as different chemical entities. Currently, a large body of research devoted to the synthesis and characterisation of these molecules is under development. ${ }^{2}$ From a surface science perspective, it is observed that some tautomeric pairs adsorbed on metals show radically different conductances, i.e., an intramolecular hydrogen transfer reaction between bi-stable bonding sites can be mapped into a two-level electronic system. This has prompted a number of single-molecule manipulation studies, mainly in the framework of scanning tunneling microscopy (STM). Additionally, the issues of tautomeric selectivity and recognition have emerged, mapping the long-standing field of chirality characterisation on surfaces. The seminal manipulation work involved naphthalocyanine tautomerisation, where $\mathrm{H}$ atom transfer at the molecule central cavity was excited by inelastic electron tunneling. ${ }^{3}$ Subsequent studies have involved mostly this class of molecules, with phthalocyanine, ${ }^{4,5} \mathrm{H}_{2}$-free-base tetraphenyl-porphyrin $\left(\mathrm{H}_{2}\right.$-TPP $){ }^{6-8}$ and porphycene ${ }^{9-12}$ as prominent examples,

\footnotetext{
a) jc.tremblay@fu-berlin.de

b) maria.blanco@ehu.es
}

as well as molecular switches based on quinone derivatives $^{13,14}$ and guanine, ${ }^{15,16}$ demonstrating that $\mathrm{H}$-transfer is not necessarily restricted to the cavity of large polyaromatic molecules.

Here, we study the tautomerisation of the most stable isomer of porphine and porphycene, which has a lower symmetry than the parent molecule because of its rectangular central cavity. ${ }^{17,18}$ The positions within the molecular central cavity of the $\mathrm{H}$-saturated and hydrogenated $\mathrm{N}$ atoms, which we will refer to as amine and imine $\mathrm{N}$ in the following, respectively, define the trans and cis tautomers as shown in the inset of Fig. 1. On copper surfaces-and on metallic substrates in general-the STM-induced H-transfer is mediated by nonadiabatic coupling (NAC) between the low-energy excited electrons or holes in the metal and the vibrational degrees of freedom associated with the $=\mathrm{N}-\mathrm{H}$ bonds breaking and formation at the amine and imine groups. While on $\mathrm{Cu}(110)$ only cis $\leftrightarrow$ cis transfers could be realized experimentally, $, 10,12$ importantly, on $\mathrm{Cu}(111)$ the trans $\rightarrow$ cis tautomerisation is accomplished. ${ }^{11}$ That is, not only the porphycene conductance but also its chemical identity can be manipulated on the latter surface. This reaction on $\mathrm{Cu}(111)$ has alternatively been realized by the application of near-infrared or ultraviolet laser pulses,${ }^{19}$ which vibrationally excite the molecule indirectly via electronic excitation of the substrate, such as to create an excited intermediate from which the tautomerisation then proceeds. The experiments on $\mathrm{Cu}(111)$ also reveal the irreversibility of the STM-induced trans $\rightarrow$ cis tautomerisation at 


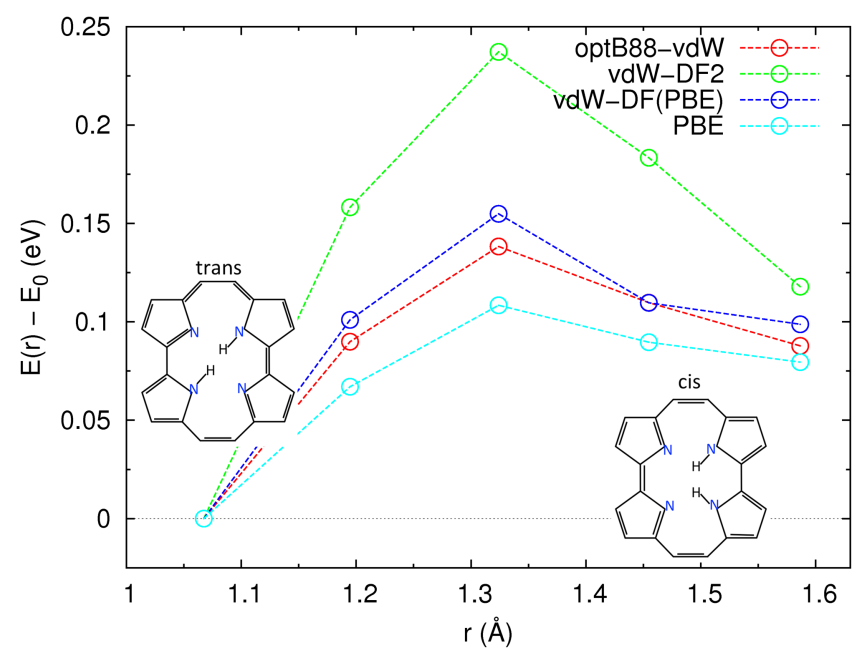

FIG. 1. One-dimensional energy barrier of the trans $\rightarrow$ cis-porphycene (shown in the insets) process in the gas phase for various functionals, referred to the potential energy $E_{0}$ of the trans tautomer, as a function of the $\mathrm{N}-\mathrm{H}$ stretched bond length $r$.

$5 \mathrm{~K}$ - the inverse process is thermally induced - which makes porphycene a good molecular switch candidate. Interestingly, the reaction can be remotely induced, i.e., it can proceed after the application of electron pulses of the STM tip located at nanoscale lateral distances from the molecule, in the low coverage regime. This suggests a likely key role of the $\mathrm{Cu}(111)$ surface state. However, this conjecture, together with other subtle details of the NAC mechanism, such as anharmonicity, intermolecular vibrational energy redistribution, and the importance of tunneling, ${ }^{20,21}$ remains open for discussion. A successful model of adsorbed porphycene tautomerisation must certainly include those ingredients, but it relies ultimately on a faithful description of the energy landscape associated with the cavity deformation, in particular the motion of the $\mathrm{N}$ and $\mathrm{H}$ atoms. Illustratively, in the STM-induced manipulation of hydrogen on/in $\mathrm{Pd}(111),{ }^{22,23}$ the potential energy topography is decisive in the dynamics description, as it was shown that a quasi-thermal mechanism promoted by NAC suffices to explain the $\mathrm{H}$-transfer behaviour under a wide range of external parameters, e.g., tunneling bias and current intensity. ${ }^{24}$

In the present work we calculate, by means of densityfunctional theory (DFT), the potential energy landscape along a one-dimensional adiabatic reaction path to describe the trans $\leftrightarrow$ cis tautomerisation of the isolated porphycene molecules adsorbed on $\mathrm{Cu}(111)$. That, in conjunction with the electronic structure analysis for both tautomers in their equilibrium configurations, serves the double purpose of (i) extracting information from the STM images (e.g., tautomer recognition, molecule buckling, and other geometrical parameters) and (ii) addressing the degree of importance of the van der Waals (vdW) interactions in the system. The latter is a fundamental aspect of the DFT study of organic and organometallic molecule adsorption on metal surfaces that, despite the latest major vdW functional developments, ${ }^{25}$ still demands benchmarking and refinement. One challenge faced in this theoretical area is functional transferability, in the sense that a functional should be valid to describe a wide range of system classes (e.g., molecular and layered crystals ${ }^{26-28}$ or molecule-metal surface interactions ${ }^{29-32}$ ) or long and short length scales (e.g., in gas-surface interactions in a dynamical environment ${ }^{33,34}$ ), simultaneously.

We find for porphycene on $\mathrm{Cu}(111)$ that there is a competition between the dispersion forces, which tend to stabilise the polyaromatic molecule ring farther from the substrate, and a significant charge exchange between the $\mathrm{Cu}$ and the cavity imine $\mathrm{N}$ atoms at closer distances. Since the combination of unsaturated $\mathrm{N}$ atoms that might serve as anchoring points ( $\mathrm{O}$ and $\mathrm{S}$ atoms, even a metal atom, could serve as well) and variable-sized polyaromatic fragments is a common feature of many organic molecules, the conclusions we draw here for porphycene can be generalised to those cases. The balance between those interactions dictates the details of the $\mathrm{H}$-transfer barrier and will, consequently, rule the inelastic scattering mechanisms driving the tautomerisation reaction. A reliable theoretical model of the process thus stems from a sensible choice of a vdW functional, all the more in the particular case of porphycene on $\mathrm{Cu}(111)$, where the small energy differences and low barriers between both tautomer conformations demand a careful account of the two aforementioned bonding contributions.

By surveying vdW functional formulations, and particularly by varying the exchange contributions, we find that several of them yield trans as the lowest energy conformation upon adsorption, as in the experiments, with energy differences of the order of $\sim 0.1 \mathrm{eV}$ only. Thus, this observation alone does not make a strong case for supporting a functional. In spite of this, we find that different functionals produce qualitative differences in the electronic structure at the interface and in the atomic coordinates (buckling of the molecule). These properties are in turn manifested as distinctive features in the simulated STM images, which allow us to further assess the functional reliability by comparison with experimental images. From this analysis we conclude that vdW-DF ${ }^{35,36}$ where PBE exchange replaces the original revPBE exchange ${ }^{37}$ offers a good overall agreement with the available experimental information on the energetics and STM images for this system.

The paper is organised as follows: the parameters for the DFT calculations and details on the vdW functionals used are reported in Section II; in Section III we show the results on the gas-phase and adsorbed porphycene energetics, and we describe the electronic structure; in Section IV we discuss those results in terms of the functionals used and interpret the experimental STM images and tautomerisation barriers. Finally, conclusions are presented in Section V.

\section{METHODOLOGY}

In the present DFT calculations, we use the periodic supercell approach to represent an infinite $\mathrm{Cu}(111)$ surface. The calculations are carried out with the VASP code, ${ }^{38}$ which uses a basis of plane waves to describe the electron wavefunctions. In the analysis of the porphycene-Cu vdW interaction, the performance of various exchange and correlation functionals is examined. We have used a few representatives of the non-local $\mathrm{DF}^{35,39,40}$ and $o p t^{40,41}$ families of DFT-vdW 
functionals, which are self-consistently ${ }^{36}$ implemented in VASP by means of an efficient algorithm to compute the dispersion corrections. ${ }^{40,42}$ The functionals of the opt family are based on the DF non-local correlation. They are made more attractive by reparameterisation of the exchange functionals $^{40,41}$ to obtain smaller deviations from the benchmark dimer data set $\mathrm{S} 22 .{ }^{43}$ As will be shown below, the attractive or repulsive character of the exchange functional employed is key to explain the relative stabilities and barrier heights of the porphycene-copper system. The literature describes how the use of alternative exchanges to those featured in the original $\mathrm{vdW}$ functional formulations improves the binding properties of several system classes. ${ }^{37,44,45}$ Therefore, we studied the role of exchange in the molecule-surface binding using vdW-DF with PBE in the semilocal exchange and correlation $\left(\right.$ outer $^{39}$ ) term $^{37}$ and vdW-DF-cx, which uses the same exchange in the outer term and in the calculation of the non-local (inner ${ }^{39}$ ) term. ${ }^{44,46}$ We have also applied other methodologies, namely Grimme's semiempirical dispersion corrections to the energy at the D3 level ${ }^{47}$ and the density-based model of TkachenkoSheffler (TkS) in its original form, ${ }^{48}$ as well as in the reparametrized version $\mathrm{vdW}^{\text {surf }} 49$ which accounts for the manybody screening of the substrate electrons, and the many-body dispersion (MBD) approach. ${ }^{50,51}$ Note that in the latter a screened polarizability in the metal substrate atoms needs to be considered. ${ }^{52}$

Finite slabs with four $\mathrm{Cu}$ layers and a $(6 \times 6)$ lateral periodicity were found to be wide enough for the lateral $\mathrm{vdW}$ interactions between molecules to be negligible. We keep the Cu lattice constant fixed to its experimental value, $a_{0}=3.61 \AA$, and we also fix the two bottom $\mathrm{Cu}$ layers, while allowing for the relaxation of all other atomic coordinates. The vacuum space between the slabs is equivalent to $7 d_{0}$, where $d_{0}$ is the bare surface interlayer spacing. Note that vdW-DF2 largely overestimates lattice constants. ${ }^{40}$ In fact, here we observe an overestimation of about $4 \%$ in the interlayer distance at the topmost layer. In this particular case, only the molecular degrees of freedom are optimized to avoid unphysical expansion of the surface. Ion cores are treated in the calculations through projector-augmented wave (PAW) potentials. ${ }^{53}$ The energy cutoff for the basis set is $400 \mathrm{eV}$ and the MonkhorstPack mesh for the first Brillouin zone $k$-point sampling ${ }^{54}$ is $3 \times 3 \times 1$. To extract the properties of the gas-phase molecule, a larger hexagonal supercell of $14 \times 14 \times 10 \AA^{3}$ and a $1 \times 1 \times 1$ $k$-point mesh was used. The convergence criteria for the total energies in the self-consistent loop are $10^{-5} \mathrm{eV}$. In the geometry optimizations, the atomic coordinates are considered converged when the energy differences are $<10^{-5} \mathrm{eV}$ and forces $<0.02 \mathrm{eV}^{-1}$.

We use constrained minimization to obtain the onedimensional tautomerisation energy barrier between the trans and cis potential energy minima. The corresponding reaction coordinate is determined by following the potential energy landscape along the $\mathrm{N}-\mathrm{H}$ distance of the hopping $\mathrm{H}$ atom. At each point along the calculated path, this distance is kept fixed (i.e., it is a nonlinear constraint) and the remaining molecular degrees of freedom are allowed to relax and follow the $\mathrm{H}$ migration adiabatically. All the structures along the reaction path were optimised with the ASE package $^{55}$ using the FIRE algorithm. ${ }^{56}$ To obtain initial guesses for the intermediate structures, the Cartesian coordinates of the trans and cis equilibrium configurations were linearly interpolated.

\section{RESULTS}

\section{A. Tautomerisation in the gas phase}

Table I shows the potential energy difference $E_{c-t}$ between the cis and trans configurations in the gas phase (a positive $E_{c-t}$ indicates that trans is more stable than cis) and the energy at the trans $\rightarrow$ cis transition state (TS) for various functionals, compared with B3LYP ${ }^{57,58}$ and MP2 $2^{59,60}$ literature values. ${ }^{61,62}$ Fig. 1 shows the one-dimensional potential energy profile of this tautomerisation process calculated with constrained minimization. The vdW-DF2 functional results in much larger $E_{c-t}$ and barrier values than the other studied functionals, which lie closer to the MP2 data which we consider a reliable reference. The best agreement is found for the vdW-DF with PBE exchange. We recall that the original vdW-DF is constructed with revPBE exchange. Note that results labelled as PBE are obtained with PBE semilocal correlation, while the vdW-DF(PBE) ones are obtained with the local density approximation (LDA) plus non-local correlation. Therefore, the differences in the energies obtained from these two levels of approximation might be attributed to the two intramolecular hydrogen bonds $\mathrm{N}-\mathrm{H} \cdots \mathrm{N}$, as the main supplementary sources of dispersion interactions. This seems to be a minor effect, though, as differences of only $\sim 20 \mathrm{meV}$ are found (see Fig. 1).

\section{B. Tautomerisation in the adsorbed configurations}

We calculate next the energy minima corresponding to the adsorbed trans and cis configurations. Since the STM exper-

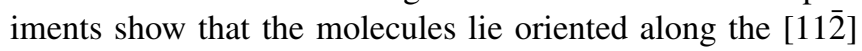
surface crystallographic direction or their symmetry equivalents (in total they can adopt six equivalent orientations and both chiralities of the isomers), in the present work we consider the configurations shown in Fig. 2, which guarantee that both unsaturated $\mathrm{N}$ atoms of the cis isomer are in registry with neighbouring $\mathrm{Cu}$ atoms. This configuration is supported by the fact that the experiments show a rigid shift of the molecule of

TABLE I. Relative energies of gas-phase trans and cis porphycene and trans $\rightarrow$ cis barrier heights $E_{T S}$ from VASP calculations (in $\mathrm{eV}$ ) for various functionals. Literature values calculated with TZ2P and 6-31G(d,p) Gaussian basis sets at the B3LYP and MP2 levels are shown, too.

\begin{tabular}{lcc}
\hline \hline & $E_{c-t}$ & $E_{T S}$ \\
\hline optB86b-vdW $^{10}$ & 0.077 & \\
optB88-vdW & 0.088 & 0.138 \\
vdW-DF2 & 0.118 & 0.237 \\
PBE & 0.080 & 0.108 \\
vdW-DF(PBE) & 0.099 & 0.155 \\
\hline B3LYP (TZ2P & & \\
B3LYP $\left(6-31 G(d, p)^{62}\right)$ & 0.104 & 0.213 \\
MP2 22 & 0.100 & 0.203 \\
\hline \hline
\end{tabular}




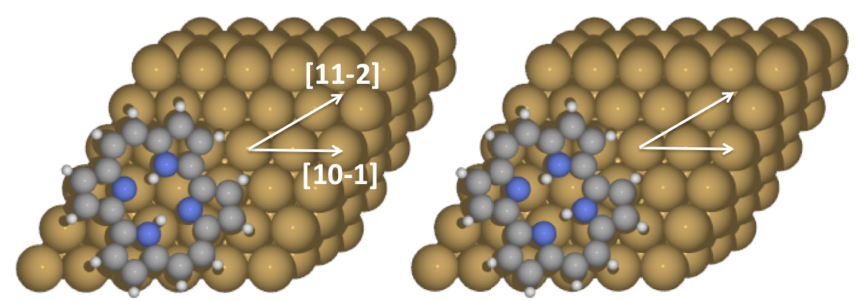

FIG. 2. Models of trans and cis-porphycene adsorption on $\mathrm{Cu}(111)$.

$\sim 1.4 \AA$ along [1110] (symmetry equivalent to [101]]) during the cis $\leftrightarrow$ cis tautomerisation. ${ }^{11}$ Although no evident conformation that pairs up the unsaturated $\mathrm{N}$ and $\mathrm{Cu}$ atoms of the $\mathrm{Cu}(111)$ substrate exists for the trans isomer, no significant shift or rotation of the molecule is observed upon trans $\rightarrow$ cis transformation. This supports the structures shown in Fig. 2 where the molecule lies at a hollow site as being the most stable ones, which is confirmed by most density functionals. Experimentally, it is difficult to distinguish $f c c$ from $h c p$ hollows or to determine the adsorption height of the molecules.

Table II summarizes the $E_{c-t}$ energies and adsorption heights, given as the average height $\tilde{z}$ of the four pyrrole centres, obtained with several functionals for Fig. 2 geometries. According to the experiment, all the molecules adsorb only in the trans conformation. The thermodynamical stability of the trans molecules proves univocally that values $E_{c-t}>0$ should be obtained. Theoretically, we observe that optB86b-vdW, optB88-vdW, and TkS (with PBE exchange) fail to reproduce this qualitative trend, yielding a cis configuration, that is, $\sim 0.15 \mathrm{eV}$ more stable than the trans one. The many-body corrections to the latter functional $\mathrm{vdW}^{\text {surf }}$ and MBD do not modify this conclusion, although they significantly reduce the energy difference between both configurations. The Grimme vdW-D3 functional also predicts the wrong ordering and about the same stability when the PBE
TABLE II. For various DFT functionals, adsorption energy difference between cis and trans configurations on $\mathrm{Cu}(111)$, and molecule heights measured as the average height $\tilde{z}$ of the centres of mass of the four pyrroles in each molecule.

\begin{tabular}{lccc}
\hline \hline Functional & $E_{c-t}(\mathrm{eV})$ & $\tilde{z}_{\text {trans }}(\AA)$ & $\tilde{z}_{\text {cis }}(\AA)$ \\
\hline optB86b-vdW & -0.130 & 2.50 & 2.65 \\
optB88-vdW & -0.167 & 2.69 & 2.72 \\
DFT-D3(PBE) & -0.115 & 2.59 & 2.54 \\
DFT-D3(revPBE) & -0.034 & 2.63 & 2.57 \\
DFT-D3(RPBE) & -0.011 & 2.61 & 2.60 \\
TkS & -0.159 & 2.59 & 2.62 \\
vdW surf & -0.081 & 2.52 & 2.53 \\
MBD & -0.093 & 2.49 & 2.55 \\
vdW-DF(PBE) & 0.085 & 3.29 & 2.96 \\
vdW-DF-cx & 0.049 & 3.29 & 2.87 \\
vdW-DF2 & 0.106 & 3.55 & 3.54 \\
vdW-DF2(PBE) & 0.094 & 3.42 & 3.43 \\
\hline \hline
\end{tabular}

exchange is used. However, the cis stability is largely reduced for other exchange choices: revPBE and RPBE result in nearly equally stable trans and cis, with absolute values of $E_{c-t} 0.034$ and $0.011 \mathrm{eV}$, respectively. We observe two typical adsorption heights for both tautomers across the table: $\tilde{z} \simeq 2.5 \AA$ for the functionals that yield energies $E_{c-t} \simeq-0.15 \mathrm{eV}$ or $E_{c-t} \simeq 0$, in disagreement with the experimental observations, and $\tilde{z} \simeq 3.5 \AA$ for the ones resulting in values $E_{c-t} \simeq 0.1 \mathrm{eV}$. The vdW-DF(PBE) and vdW-DF2 functionals provide similar $E_{c-t} \simeq 0.1 \mathrm{eV}$, consistent with the experimental result regardless of the choice of the exchange functional. The behaviour of vdW-DF-cx is similar to vdW-DF(PBE), with a slightly more strongly bound cis. They differ significantly, though, in the height and shape of the tautomerisation barrier, as shown in Fig. 3 top panel, as well as in the distance $\tilde{z}$ of cis-porphycene to the surface. The original vdW-DF2 functional, which uses
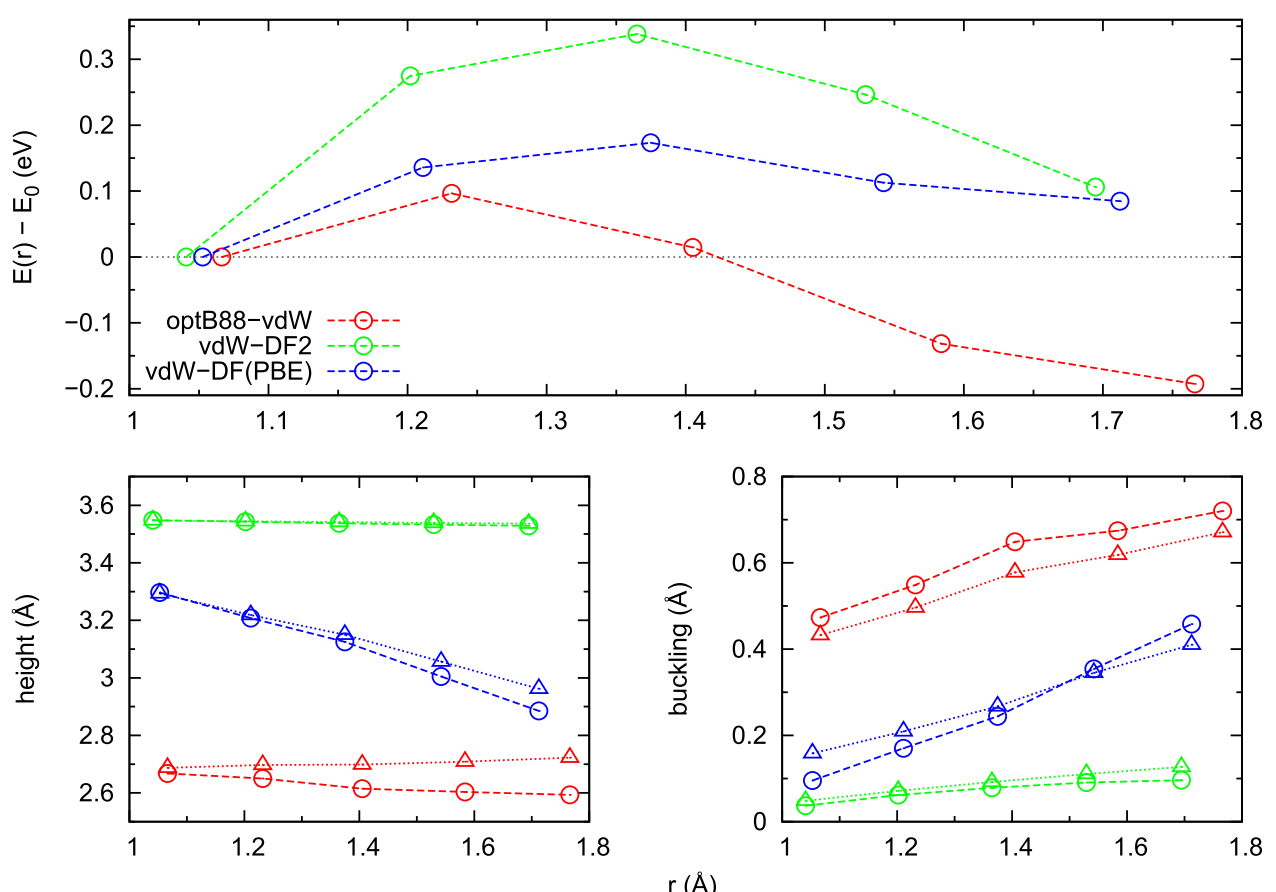

FIG. 3. Top panel: trans $\rightarrow$ cis onedimensional barriers for porphycene adsorbed on $\mathrm{Cu}(111)$ for several functionals, referred to the potential energy $E_{0}$ of the trans tautomer, as a function of the $\mathrm{N}-\mathrm{H}$ stretched bond length $r$. Bottom left and right panels: change in the average heights above the topmost $\mathrm{Cu}$ layer and bucklings, respectively, of the molecules during $\mathrm{H}$-transfer. Circles and triangles show the quantities as average values of the four $\mathrm{N}$ atoms or the four pyrrole centres, respectively. 


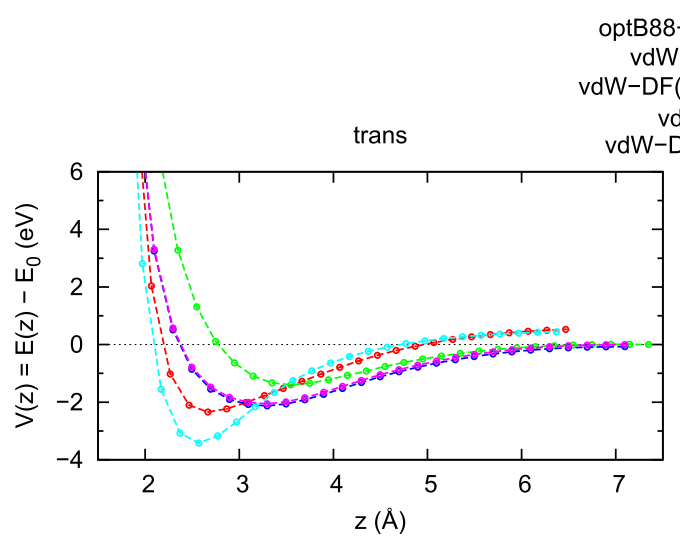

optB88-vdW ---o--.

$\mathrm{VdW}-\mathrm{DF} 2$

dW-DF(PBE) --o-vdW $^{\text {surf }}$

cis

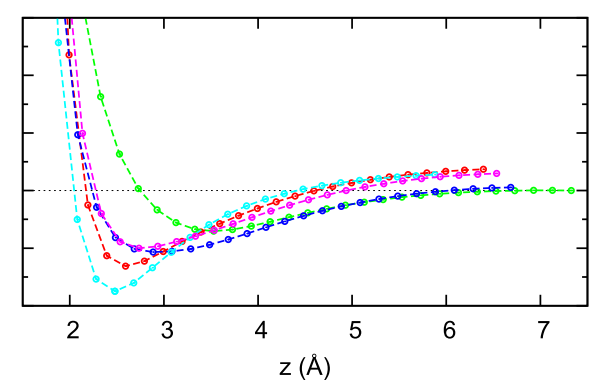

FIG. 4. One-dimensional adsorption potential for porphycene approaching the $\mathrm{Cu}(111)$ surface. The minimum corresponds to the cis and trans minima shown in Fig. 3. The $z$ values represent the molecule height (measured as the average height of the $\mathrm{N}$ atoms) for equispaced rigid shifts of the unrelaxed molecule. The energy zero of each potential curve indicates the adsorption energy $E_{0}$ with respect to the relaxed gas-phase molecular geometry.
rPW86 exchange, overestimates the experimental cis $\rightarrow$ trans barrier of $42.3 \pm 2.7 \mathrm{meV}$, while vdW-DF(PBE) is in a good agreement with the experiment (the functional vdW-DF-cx also overestimates the barrier, mainly because the cis conformation is more stable). In this figure and in the analysis hereafter, we focus on the three functionals that are the representative of the different DFT-vdW functionals and moleculesubstrate interaction strength scenarios: the strongly attractive optB88-vdW, the weakly attractive vdW-DF2, and the vdW$\mathrm{DF}(\mathrm{PBE})$ as an intermediate case. The medium strength of the latter functional is also revealed in the adsorption heights $\tilde{z}_{\text {trans }}=3.29$ and $\tilde{z}_{\text {cis }}=2.96 \AA$, which are intermediate between the two typical heights mentioned above (see Table II). The binding strength provided by each of these functionals is reflected in the variation of the molecular structure as $\mathrm{H}$ is being transferred from one $\mathrm{N}$ atom to the other. This is shown in the bottom panels of Fig. 3, where the adsorption heights and molecule bucklings are given as the averages of the heights of the four $\mathrm{N}$ atoms $(z)$ and of the pyrrole centres $(\tilde{z})$ above $\mathrm{Cu}(111)$. These two ways of accounting for the geometrical details provide very similar information, with differences of less than $0.1 \AA$. These could be attributed to the distortion of the polyaromatic ring along the reaction path, which is barely observable with the bare eye. The overbinding character of optB88-vdW is reflected in the small adsorption heights and larger buckling of the molecule. The opposite behaviour is obtained for the weakly binding vdW-DF2. In the intermediate vdW-DF(PBE) case, we observe a progressive decrease in the height and an increase in the buckling along the reaction path.

The molecule adsorption energies $E_{a d s}$ with respect to the gas-phase and the attraction range exerted by five of the studied functionals are depicted in the molecule-surface potential energy as a function of the molecule height, $V(z)$, in Fig. 4. The curves are calculated by shifting the rigid molecule upward and downward from the potential energy minima configurations, i.e., the relative atomic positions are not relaxed. No barrier for the molecule approach from the gas phase is observed, at least with these constraints. The energy zero in each curve is taken at the potential energy of the molecule lying far from the clean surface in its relaxed configuration, i.e., the energy value at the minimum is $E_{a d s}$. Because of the constraints adopted here, some of potential curves at high $z$ values tend to $\sim 0.7 \mathrm{eV}$ due to the strong buckling caused by adsorption near the surface. We observe $E_{a d s}$ differences of the order of $1 \mathrm{eV}$ depending on the functional and wider adsorption wells for the least binding ones.

\section{Electronic structure of the porphycene-Cu(111) interaction}

Fig. 5 shows the sum of projected densities of states (PDOS) on the atomic orbitals of $\mathrm{N}, \mathrm{C}$, and $\mathrm{H}$, which provide a simple tool to univocally identify the binding energies of the highest occupied molecular orbital (HOMO) and lowest unoccupied molecular orbital (LUMO) in particular for a weak hybridisation with the surface. This is clearly the case for vdW-DF2 and for trans-porphycene with vdW-DF(PBE), where the HOMO and LUMO peaks are localised. In the other cases, the peaks are less clear as a consequence of a significant hybridisation between the MOs and the $\mathrm{Cu}(111)$ substrate. To confirm this interpretation, we have also inspected the KohnSham (KS) states located at the relevant peaks or energy ranges in the PDOS curves and compared them with the KS states calculated in the gas-phase that correspond to the HOMO and LUMO (see the charge density associated to the gas-phase

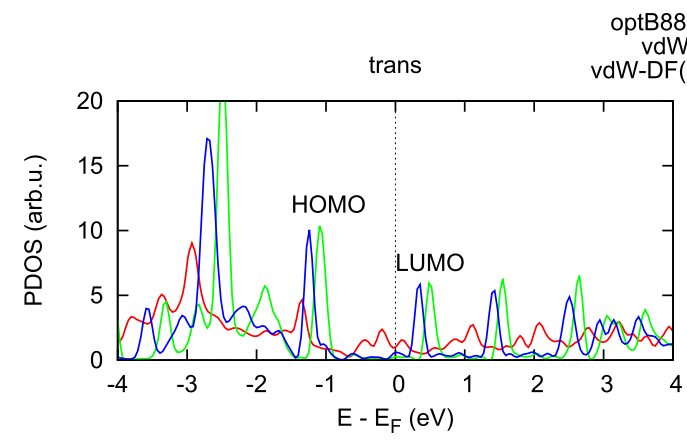

vdW-D8-vdW
vdW-DF2 dW-DF(PBE) cis

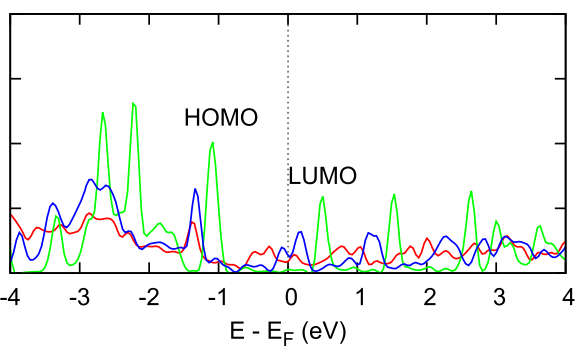

FIG. 5. PDOS of cis and transporphycene near the Fermi level for various functionals. 
TABLE III. Charges $\Delta q$ in $|e|$ units of the adsorbed molecules relative to their gas-phase nominal values calculated from a Bader partitioning scheme of the charge inside the calculation supercell. A negative (positive) sign corresponds to the electron transfer from (to) the $\mathrm{Cu}$ surface to (from) the molecule.

\begin{tabular}{lrr}
\hline \hline Functional & trans & \multicolumn{1}{c}{ cis } \\
\hline optB88-vdW & -0.550 & -0.576 \\
vdW-DF2 & 0.039 & 0.037 \\
vdW-DF(PBE) & 0.054 & -0.243 \\
\hline
\end{tabular}

MOs in Fig. 7). For the optB88-vdW and vdW-DF(PBE) functionals, the cis-porphycene hybridisation with the $\mathrm{Cu}$ substrate is strong enough to shift the LUMO downward in energy around the Fermi level. Only for the most attractive functional, does the trans LUMO hybridise close to the Fermi level. The HOMO lies well below, at about $-1 \mathrm{eV}$.

The HOMO-LUMO gap of both isomers in the gas phase takes an approximate value of $1.6 \mathrm{eV}$ for the investigated functionals (the experimental gas-phase value is $2.2 \mathrm{eV}^{63}$ ). Fig. 5 shows that the gap is not significantly altered upon adsorption, but marginal changes due to screening effects might be present. Although we cannot expect accurate gap values at this level of theory, we can infer charge transfer differences because of the functional-dependent gap alignment with respect to the Fermi level. The use of the hybrid functionals, which include exact exchange, in combination with a nonlocal correlation correction could reduce selfinteraction errors and thus improve the quantitative description of the HOMO and LUMO binding energies, as well as of the charge transfer. ${ }^{64}$ These calculations currently come at a prohibitive computational cost for a system like the present one. We have determined the actual charge transfers from a Bader-like atoms-in-molecules charge partitioning analysis. ${ }^{65}$ Table III shows the charge depletion or accumulation $\Delta \rho$ at the adsorbed molecule with respect to the gas phase. Bear in mind that a Bader analysis of our calculated gas-phase porphycene electron densities underestimates the nominal total charge in the molecule by 0.13 electrons. Thus we can consider this value as an error bar for the $\Delta \rho$ values of Table III. As expected, vdW-DF2 yields negligible and optB88-vdW yields the largest charge transfers. In the latter case, about 0.6 electrons are donated by the surface to both isomers. The third functional yields a significant donation to the cis but not to the trans porphycene. All these features of the Bader analysis are consistent with the results of the PDOS presented in Fig. 5.

The charge density alterations at the interface are studied in further detail with the so-called charge density difference (CDD) map, i.e., the difference between the actual electron density in the adsorbed configuration and the sum of the bare surface and the isolated molecule densities, both calculated with the atomic positions frozen at the adsorbed configuration geometry. Fig. 6 shows two-dimensional cuts of the CDD perpendicular to the surface through the imine $\mathrm{N}$ atoms. There it can be distinguished how bonds are formed between these $\mathrm{N}$ atoms and some substrate $\mathrm{Cu}$ atoms, with a quite clear directionality in some of the cases. This happens mainly for optB88-vdW and both imine $\mathrm{N}$ atoms of cis and also one $\mathrm{N}$ in trans porphycene, but also for vdW-DF(PBE) and the cis isomer. In the other cases, the much weaker bonding is manifested as a delocalised and very subtle electron accumulation at the interface. Regarding the relationship between the charge transfer and geometry, note that those directional bonds make the half portion of the cis molecule containing the imine $\mathrm{N}$ atoms that lie closer to the surface, while the other half of the molecule lies slightly higher by the influence of the vdW forces, which tend to stabilise the molecule farther from the substrate. As a matter of fact, even a small depletion of electrons is found under the amine $\mathrm{N}$ atoms of the cis molecule with optB88-vdW (not shown).

It is clear from Fig. 5 that a STM image taken at positive voltages below $\sim 1 \mathrm{~V}$ or even small negative voltages will map the molecular LUMO. Fig. 8 shows the calculated partial charge density (PCD) $2 \AA$ above the adsorbed molecules at the experimental bias voltage $-0.1 \mathrm{~V},{ }^{11}$ which can be interpreted as a coarse approximation to simulating the STM images. For a given potential bias $V$, the PCD is constructed by integration of the electron densities of the KS states lying between the Fermi level energy $E_{F}$ and $E_{F}+e V$.

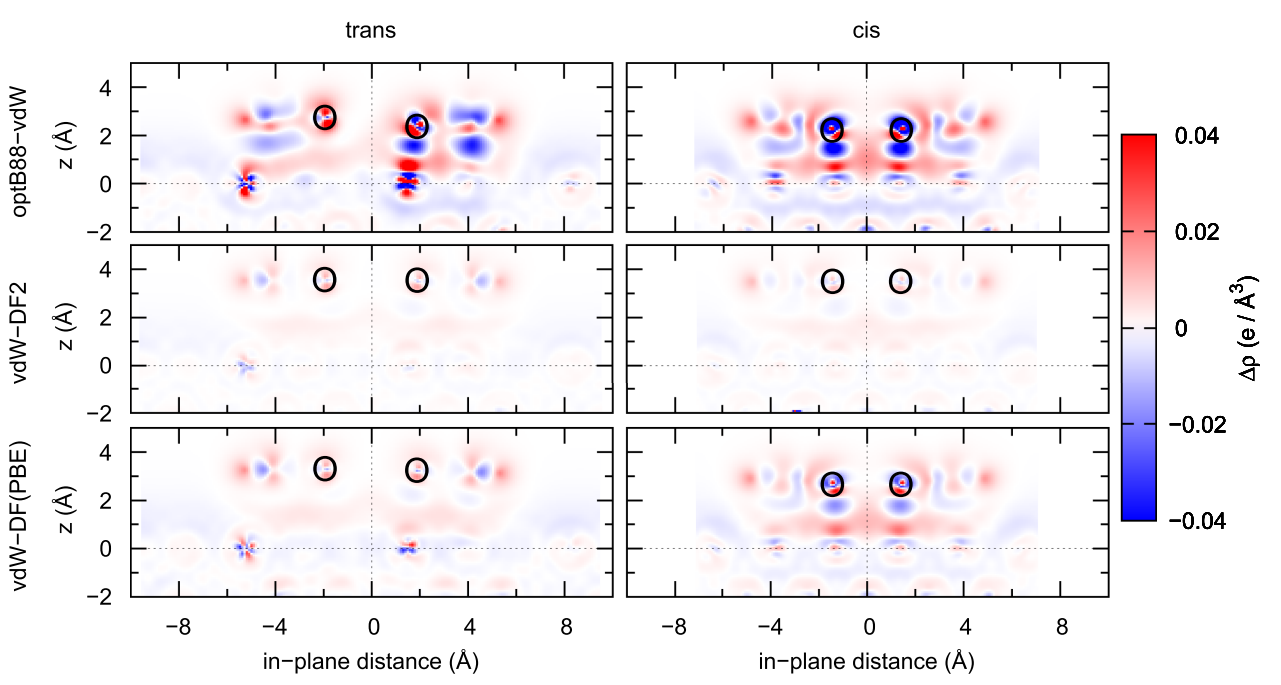

FIG. 6. Charge density difference maps of adsorbed trans and cis-porphycene for various functionals. The map is drawn at a plane perpendicular to the surface containing the two empty $\mathrm{N}$ atoms. Positive (negative) values indicate regions of electron accumulation (depletion) upon adsorption. The horizontal dashed line shows the average $z$ value of the topmost $\mathrm{Cu}$ layer and the black circles show the $\mathrm{N}$ atom positions. 


\section{DISCUSSION}

\section{A. Molecule-substrate interaction}

The original vdW-DF2 formulation, with rPW86 exchange contribution, ${ }^{66,67}$ correctly predicts the thermodynamical stability of the trans isomer upon adsorption on $\mathrm{Cu}(111)$ [as it will be discussed below, despite correctly predicting the sign of $E_{c-t}$, vdW-DF2 does not correctly describe the porphycene/ $\mathrm{Cu}(111)$ interaction], unlike optB86b-vdW and optB88-vdW, which yield a much more stable cis configuration by nearly $0.15 \mathrm{eV}$. The latter two functionals use optimised exchange contributions, formulated as the gradientmodified Becke86 ${ }^{68}$ and Becke88, ${ }^{69}$ respectively, but with a reparameterisation of the exchange enhancement factor $F_{x}(s)$ (where $s \propto|\nabla \rho| \rho^{-4 / 3}$ is the reduced density gradient and $\rho$ the point electron density) optimised to better match the S22 benchmark dataset. ${ }^{43}$ PBE and revPBE also use an alternative parametrisation of the Becke expression for the exchange enhancement factor. ${ }^{70}$ Figures 2 of Ref. 67, 2 of Ref. 40, 3(a) of Ref. 71, and 11 of Ref. 46 nicely summarize the $F_{x}(s)$ values of a number of exchange functionals in the literature. The rationale behind those formulations is that $F_{x}(s)$ must increase steeply enough with $s$ to avoid the overbinding brought by LDA, in some cases while attempting also to satisfy the LiebOxford bound ${ }^{72}$ at large $s$. This typically causes a stronger binding behaviour for PBE than for revPBE, since the latter takes larger $F_{x}(s)$ values. In order to improve in the S22 benchmarkings, RPBE, rPW86, optB86b, and optB88 are generally made to adopt intermediate values between revPBE and PBE with the following exceptions: (i) optB86b and optB88 are smaller than PBE at around $s<3$ and increase above revPBE rapidly at around $s>4.5$, (ii) rPW86 is higher than revPBE at low $s<1.8$, and (iii) RPBE and revPBE take very similar values at $s<2.5$. The overall quality of the physical description is highly dependent on the different $s$-values spanned by the electron density in each particular system. In fact, Fig. 4 shows that both tautomers are most strongly bound by optB88-vdW and least by vdW-DF2 with rPW86, while vdW-DF(PBE) shows an intermediate strength, which is reflected both in the energy and the adsorption heights. This suggests that the porphycene- $\mathrm{Cu}(111)$ interaction is mainly influenced by low- $s$ regions. This is further supported by the result of vdW-DF-cx. This functional has $F_{x}(s)$ similar to rPW86 at large $s$ values. Note that it is not directly comparable to the other studied methods of the DF family because the same exchange enters also in the nonlocal correlation term. As for the trans vs. cis difference, it is not straightforward to anticipate the effect of each functional on the relative binding energy $E_{c-t}$ and height. The results of Table II for optB88-vdW and optB86b-vdW ( $E_{c-t} \simeq-0.15 \mathrm{eV}$, in disagreement with the experiment) show that the cis tautomer is more overbound than the trans one. This makes sense if we assume that the general effect produced by these functionals is to create a strong bond at each molecule-surface anchoring point, since cis has two of these imine $\mathrm{N}-\mathrm{Cu}$ bonds and trans just one (see Fig. 6). In contrast, the two vdW-DF2 results with rPW86 and PBE exchanges [and also vdW-DF(PBE)], favour the trans tautomer by $E_{c-t} \simeq 0.1 \mathrm{eV}$ and adsorb the molecules $\sim 1 \AA$ farther from the surface.

The trend predicted by the low- $s$ enhancement factors is also observed with the semiempirical vdW-D3 functional, where PBE yields an overbound cis (same for TkS with PBE). This is in contrast with RPBE and revPBE, which yield similarly small $E_{c-t}$ values, well within the uncertainty of density functional theory. Note that the use of PBE exchange in the vdW-DFT functionals does not result in overbinding. This proves that, despite the exchange being a relevant factor in the adsorption strength, the correlation formulation is by no means less relevant to describe the dispersion interactions accurately. From these results, we infer that the above described exchange effects affect mostly the binding through the imine $\mathrm{N}$ atoms (i.e., the region of the interface below the molecule), while the outer polyaromatic ring of the molecule weakly binds to the surface. The latter is dominated by attractive dispersion forces, which tend to bind the molecule at higher $z$ values. These forces may counteract an eventually overestimated imine

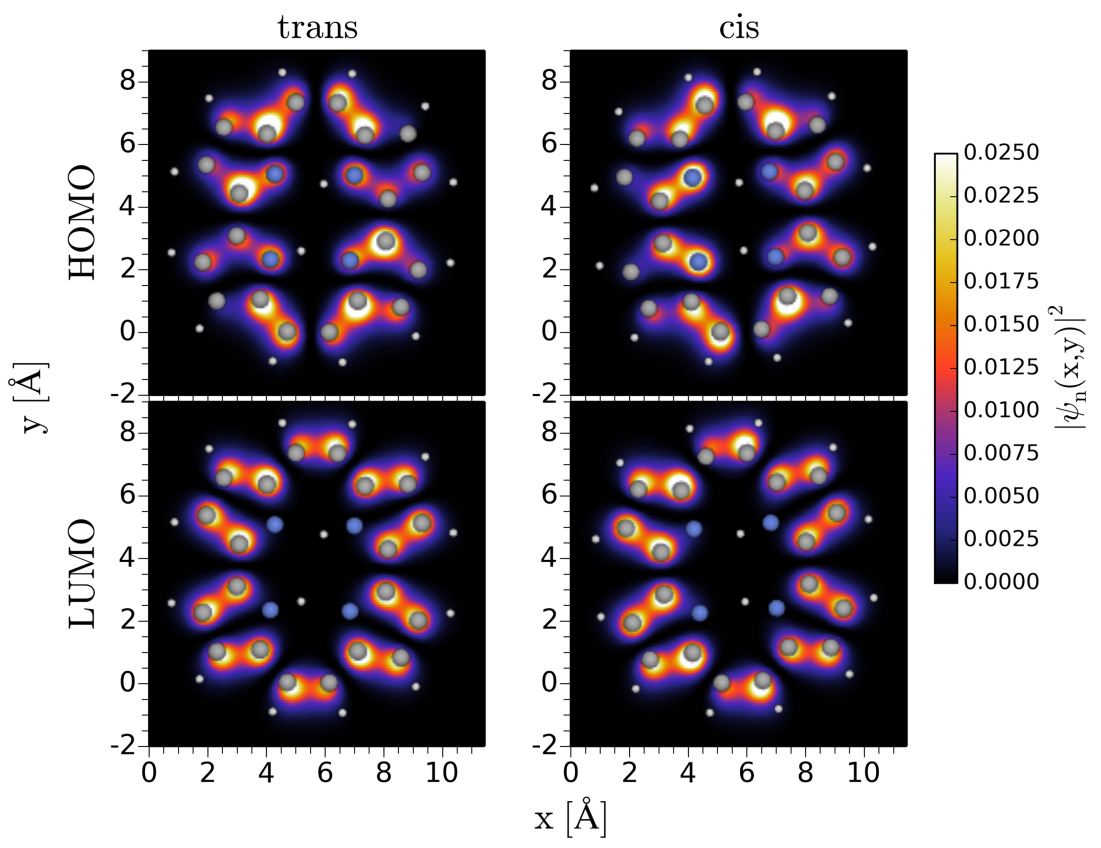

FIG. 7. Electron density associated to the HOMO and LUMO of trans and cis-porphycene. $\mathrm{H}, \mathrm{C}$, and $\mathrm{N}$ atoms are shown as white, gray, and blue balls, respectively. 
$\mathrm{N}-\mathrm{Cu}$ bond strength, causing an overall upward shift of the molecule, as we discuss in Subsection IV B.

\section{B. Electronic structure and STM interpretation}

We have analysed the electronic structure of the trans and cis adsorbed configurations for the three selected functionals. OptB88-vdW can be readily ruled out for this system on the basis of wrongly predicting that the cis tautomer is the most stable on $\mathrm{Cu}(111)$. By interpreting the experimental STM images aided by the PDOS and PCD results, we now further argue that vdW-DF2 is neither suitable.

The trans and cis STM experimental images possess distinctive patterns, which enable tautomer recognition. The double-lobed asymmetric elongated image is attributed to trans, while the crescent-shaped one to cis. This assignment can be made just by the consideration of the absence or presence of mirror symmetry (i.e., chiral or achiral character), respectively, of the gas-phase molecules. However, the knowledge of the gas-phase electronic structure alone does not grant the distinction. It is to be expected that either the HOMO or LUMO of the molecule is being imaged in the STM experiment, with some degree of blurriness associated with orbital hybridisation at the surface. In Fig. 7, both the MOs are found to look very similar for both the gas-phase tautomers, since their electronic density is localised at the polyaromatic ring. In the molecular cavity region, where the main difference (the positions of the imine and amine $\mathrm{N}$ atoms) between trans and cis resides, the changes are marginal. Therefore, we conclude that the distinctive features of the STM images must have their origin in the interaction of the HOMO and LUMO with the substrate. This finding already suggests that the very weakly binding vdW-DF2 might not be a suitable functional for modelling porphycene on $\mathrm{Cu}(111)$ either, since the molecule retains its gas phase structure and is adsorbed far from the surface $(\sim 3.5 \AA)$ for both the cis and trans structures. Another important conclusion is that the STM image modulations account for the buckling of the polyaromatic ring, which is captured by optB88-vdW and vdW-DF(PBE) only (see bottom right panel of Fig. 3).

The PDOS in Fig. 5 show that the LUMO is the closest lying orbital above the Fermi level, and that it is strongly hybridised with the surface electron density for trans with optB88-vdW and for cis with optB88-vdW and vdW$\mathrm{DF}(\mathrm{PBE})$. In these cases, the hybridised states conform to the observed STM images at low bias voltage. The interpretation of the crescents of the cis images thus appears clearly: the half of the molecule containing the amine $\mathrm{N}$ atoms is lifted, while the other one lies closer to the surface. The trans case is less clear, though, as none of the performed calculations yield enough hybridisation as to bring the imine $\mathrm{N}$ atoms closer to the surface and produce the needed asymmetry in the PCD, with the exception of opB88-vdW. A buckling with the same origin has been reported for $\mathrm{H}_{2}-\mathrm{TPP},{ }^{7}$ which does not seem to be the case here. The brightest lobes were interpreted in the experimental literature as closely lying to the axis along the molecule diagonal that crosses the amine $\mathrm{N}$ atoms, revealing the molecule handedness. ${ }^{11}$ This seems to be a natural
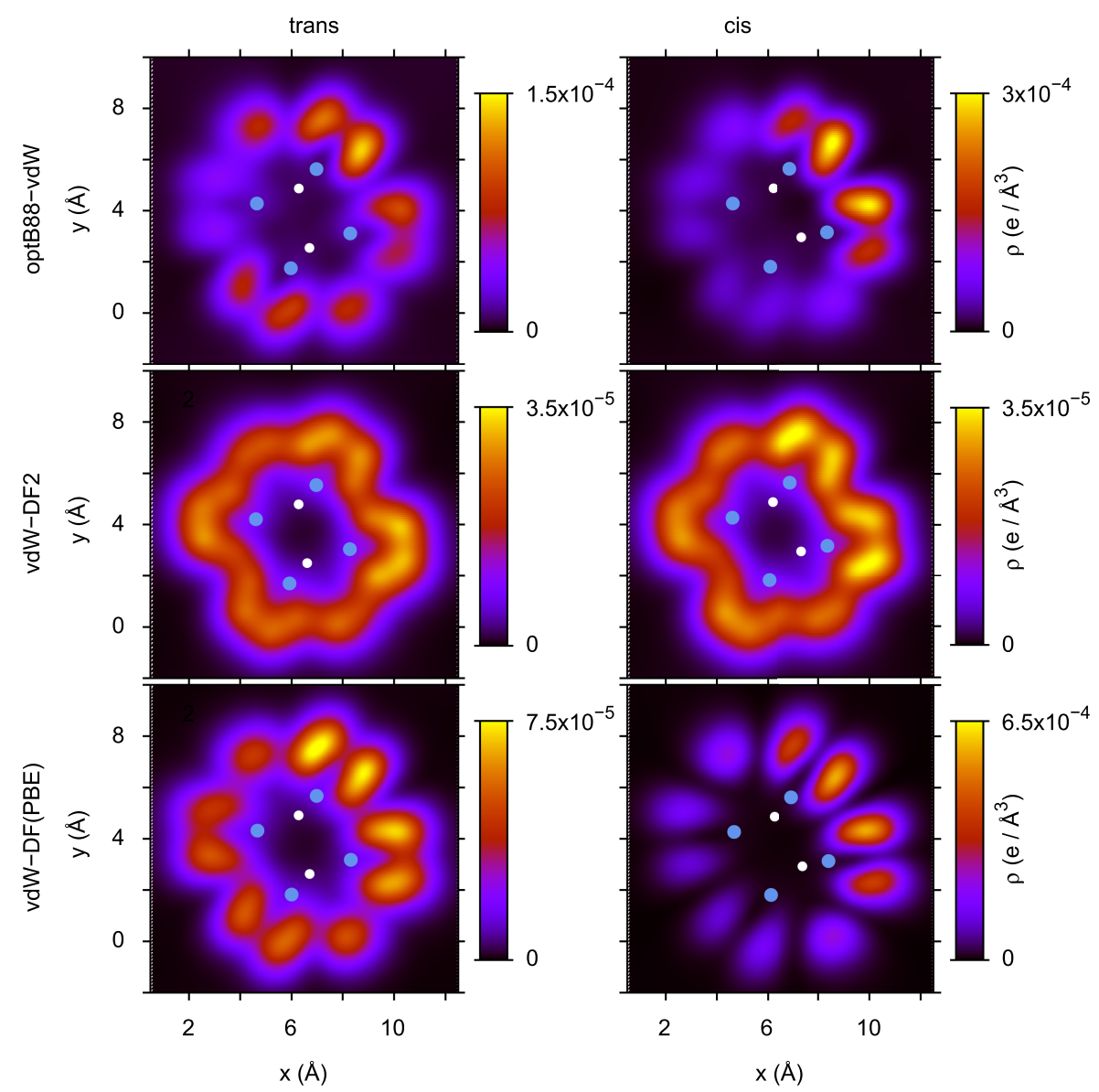

FIG. 8. Partial charge density cut at $z$ $=2 \AA$ Above the molecule of Kohn-Sham states integrated in an energy window of $0.1 \mathrm{eV}$ below the Fermi level (i.e., the bias voltage used in the images of Ref. 11 for the trans and cis-porphycene isomers) obtained with three functionals. The $(x, y)$ coordinates of the cavity $\mathrm{N}$ and $\mathrm{H}$ atoms are indicated by large blue and small white circles, respectively. 
interpretation, as the unsaturated $\mathrm{N}$ atoms lie closer to the surface when vdW-DF(PBE) and especially optB88-vdW are used. The PCD at $V=-0.1 \mathrm{~V}$ (see Fig. 8) for these two functionals shows that there is enough hybridisation as to produce the modulation of the LUMO needed to reproduce the asymmetric lobes and the crescent shape characteristic of the trans and cis tautomers, respectively. Note that the vdW-DF(PBE) seems to slightly underestimate the hybridisation, producing just a weak asymmetry. Fig. 5 demonstrates the weakness of the surface interaction with both tautomers for vdW-DF2, which is reduced to a pure $\mathrm{vdW}$ interaction contribution at long distances $\sim 3.5 \AA$ with no charge exchange and very little molecular buckling. As expected, the simulated images (Fig. 8) are in a qualitative disagreement with the experimental ones because the PCD at $V=-0.1 \mathrm{~V}$ does not include any MO character at all. Therefore, we argue that vdW-DF2 should be disregarded, too. From the buckling calculated with vdWDF(PBE) and optB88-vdW (see Fig. 3), and the experimental images, we may deduce that a buckling of $\sim 0.2 \AA$ produces a small, yet noticeable, modulation in the STM images, and that bucklings above $0.4 \AA$ result in very clear contrasts. Note that, here, no attempt was made to enhance the contrast of the PCD images and the STM response is depicted as a linear scale.

The Bader analysis (see Table III) allows to quantify the hybridisation in terms of the charge $\Delta q$ transferred from the substrate to the molecules. As expected, $\Delta q$ is negligible for vdW-DF2. However, we note that the porphycene-Cu binding energies do not correlate with the degree of charge transfer. In fact, optB88-vdW stabilises cis more than trans on $\mathrm{Cu}(111)$, but yields similar $\Delta q \simeq-0.55|e|$ in both cases. For the medium-binding vdW-DF(PBE), we find negligible $\Delta q$ for trans and $\Delta q \simeq-0.25|e|$ for cis, consistent with the LUMO hybridisation features observed in the PDOS of Fig. 5. Similarly, there is a clear correlation between large $\Delta q$ values and low adsorption heights but not between large $\Delta q$ and $E_{c-t}>0$ values. The CDD maps of Fig. 6 show that the strongly directional $\mathrm{Cu}$-imine- $\mathrm{N}$ bonds are the main contributors to the reported $\Delta q$ values. There, we also observe that in the optB88-vdW case the interaction with $\mathrm{Cu}$ causes a significant charge rearrangement within the molecule, not only in the interface region. This extra localised charge redistribution, probably conditioned by the underestimation of the molecule adsorption height, has an important effect on the interaction energetics. It thus appears as one of the main factors responsible for the wrong energetic ordering [cis more stable upon adsorption on $\mathrm{Cu}(111)]$ using this functional.

The degree of charge exchange between the substrate and the single molecule is relevant for understanding the stability of porphycene at higher coverages. This correlation with lateral interactions between molecules, which add to the expected usual weak lateral vdW contribution, has been reported for 2H-porphine on $\mathrm{Ag}(111)$ and $\mathrm{Cu}(111) .^{73,74}$ Although the characterisation of coverage effects lies beyond the scope of this work, it is worthwhile pointing out that lateral interactions are expected to be weak in the present case. As a worst case scenario, let us consider the vdW-DF(PBE) functional, for which the value for the charge transfer varies from $\Delta q=-0.243|e|$ at $z=2.96 \AA$ above the surface in the cis case to $\Delta q=0.054|e|$ at $z=3.29 \AA$ in the trans case. Creation of an image charge in the surface will lead to the creation of a dipole, $\mu_{\mathrm{CT}}=2 \Delta q z$. The dominant contribution to the lateral interactions can be evaluated as a simple dipole-dipole coupling term between two molecules in the adjacent cells $(R=15.3 \AA)$. In the present case, this amounts to an energetic correction $\left(E_{\mathrm{CT}}=-\frac{\mu_{\mathrm{CT}} \mu_{\mathrm{CT}}}{R^{3}}\right)$ of the order of $8 \mathrm{meV}$ and $0.5 \mathrm{meV}$ for the cis and the trans conformers, respectively. This will not affect their relative stability and, consequently, we will neglect them in the following. Further, any investigation of the coverage dependence would benefit from a functional that gives a good account of the amount of hybridization, which is the aim of this paper.

\section{Tautomerisation energetics and geometry}

The trans $\rightarrow$ cis transition on $\mathrm{Cu}(111)$ can be either induced by inelastic electron tunneling ${ }^{11}$ or by photoexcitation $^{19}$ at low temperature. The reverse reaction, however, does not proceed from these principles. Instead, if light or electron pulses are applied to the adsorbed cis monomers, a double $\mathrm{H}$ transfer, the cis $\leftrightarrow$ cis reaction, takes place. The cis $\rightarrow$ trans process is thermally achieved upon heating the surface up to $30-35 \mathrm{~K}$. These results in all the cis molecules are being transformed back to the trans conformer. The barrier of this activated process is determined to be $42.3 \pm 2.7 \mathrm{meV} .{ }^{11}$ From vdW-DFT (see Fig. 3), we obtain the best agreement in this reverse barrier for vdW-DF(PBE), with $89 \mathrm{meV}$, whereas the other functionals largely overestimate it. We observe here that the functionals do not just affect the equilibrium configurations energetics but, very importantly, also the transition states.

Interestingly, the barriers for both forward and backward $\mathrm{H}$-transfer processes are higher than the gas-phase ones, i.e., the surface has a stabilising effect on the tautomers such that no catalysing effect is observed. Intuitively, this makes sense because the $\mathrm{N}-\mathrm{H} \cdots \mathrm{N}$ bonds that need to be formed and broken compete with the breaking of the $\mathrm{Cu}$-imine $\mathrm{N}$ bonds described above. This seems to be the case of vdW-DF(PBE) and, more clearly, of the optB88-vdW cis $\rightarrow$ trans process. Therefore, applying this same argument to the vdW-DF2 case would lead us to expect similar energetics as in the gas phase. However, this is not the case, and indeed we observe that the TS lies $\sim 0.1 \mathrm{eV}$ higher than in the gas phase. This unphysical behaviour, in addition to the disagreement with the gas-phase MP2 data, ${ }^{62}$ further dissuades from using vdW-DF2 in this system.

The functional choice also affects the molecule atomic coordinates during $\mathrm{H}$-transfer, as shown in the bottom panels of Fig. 3. The competition between hybridisation and $\mathrm{vdW}$ interaction results in the buckling of the molecule, as each of these contributions tends to stabilise the adsorbates at different distances from the metal, typically in the ranges of 2.5 and $3.5 \AA$, respectively. The height-dependent potentials of Fig. 4 reflect this, too. Since the three functionals contain similar non-local correlation contributions, they have a similar attractive character at long ranges $(z \geq 4 \AA)$. This is additionally confirmed by similar $V(z)$ curves calculated using the exact same geometries (not shown). The decisive contribution to the binding is made by the exchange part at lower ranges ( $z \leq 2.2 \AA$ ), where optB88-vdW is the most repulsive, while 
vdW-DF2 becomes equally repulsive already at $z \simeq 2.7 \AA$. The vdW-DF(PBE) curve, which lies close to the optB88-vdW one at low $z$, shows that the use of PBE exchange helps suppress the short-range repulsion. ${ }^{37}$ For the trans molecule vdW-DF-cx follows closely that of vdW-DF(PBE), while a slightly different behaviour is observed for cis. Finally, note that from the five functionals shown in Fig. 4, vdW ${ }^{\text {surf }}$ is clearly the most strongly binding one.

Although the onset of the trans $\rightarrow$ cis tautomerisation is observed at a $150 \mathrm{mV}$ bias, a value close to the forward $\mathrm{vdW}-\mathrm{DF}(\mathrm{PBE})$ barrier, the results here obtained cannot explain by themselves the irreversibility of the STM or photoinduced processes. For that, we should bear in mind the large number of other factors (non-adiabatic couplings, intramolecular vibrational energy redistribution, etc.) involved in the reaction beyond the plain potential energy topography along the reaction path, which are far from the scope of this work. Finally, in Ref. 11 it is proposed that the $\mathrm{Cu}(111)$ surface state electrons enhance the cis yields by manipulation with STM. Under this assumption, the obtention of reliable adsorption height values with vdW-DFT becomes increasingly important, as height variations can dramatically change the scattering of the bidimensionally localised metal electrons at the adsorbate, as it has been observed in $\mathrm{H}_{x}$-TPP $(x=\{0,1,2\})$ molecules on $\mathrm{Au}(111),{ }^{8}$ for example.

\section{CONCLUSION}

We have studied by DFT the adsorption of trans and cis porphycene on $\mathrm{Cu}(111)$ and calculated the energy barrier for the associated intramolecular $\mathrm{H}$-transfer known as tautomerisation. Experimentally, trans $\leftrightarrow$ cis at low temperature can be irreversibly photo-induced with UV lasers ${ }^{19}$ or electroinduced using the pulses from a STM tip, ${ }^{11}$ and the reverse process is thermally activated. The issues of irreversibility and other manipulation details are of dynamical nature originating from non-adiabatic coupling, and they will be the subject of a future publication. Since the energetics of this type of systems are extremely sensitive to the balance between dispersion forces and exchange contributions, the scope of the present work was to determine the proper choice of functional.

From calculations that use the non-local vdW-DF functional ${ }^{35}$ coupled with PBE exchange, we find the trans tautomer to be more stable than cis, as it happens in the gas phase, by $E_{c-t}=0.085 \mathrm{eV}$ and a trans $\rightarrow$ cis barrier of $0.173 \mathrm{eV}$, in reasonable agreement with the experiments. ${ }^{11}$ The barrier is $0.054 \mathrm{eV}$ higher than the gas-phase one, which reveals the stabilising effect of the $\mathrm{Cu}$ substrate. Unlike on $\mathrm{Cu}(111)$, on $\mathrm{Cu}(110)$ trans species are reported to be extremely short-lived $^{12}$ and only cis $\leftrightarrow$ cis transformations, probably by concerted transfer of both $\mathrm{H}$ atoms, are observed. ${ }^{9,10,12}$ DFT calculations in the literature yield $E_{c-t}=-0.19 \mathrm{eV}$ for $\mathrm{Cu}(110),{ }^{10}$ while only trans tautomers and trans $\leftrightarrow$ trans transitions are observed on the polymeric substrates. ${ }^{75}$ This confirms that the substrate plays a fundamental role in tautomerisation reactions in porphycene, both in selectivity and catalysis.

Porphycene adsorption on $\mathrm{Cu}(111)$ is the result of two contributions: van der Waals forces are responsible for the binding of the outer polyaromatic cycle of the molecule to $\mathrm{Cu}(111)$, and the molecule anchors to individual $\mathrm{Cu}$ atoms via the unsaturated $\mathrm{N}$ atoms located at the molecule central cavity. In the electronic structure, this is reflected as a partial hybridisation of the LUMO with the metallic states at the Fermi level, which produces the characteristic features of each tautomer in the STM images. In our analysis we attribute these features to the molecule buckling, which is enhanced for the lowerlying cis tautomer. After analysing various representatives of functional families, we find that a correct DFT description of the balance between those two mechanisms requires a sensible choice of the exchange formulation. On the one hand, optB88-vdW ${ }^{41}$ is found to be too attractive because it overestimates LUMO hybridisation and predicts that the cis conformer is the most stable $\left(E_{c-t} \simeq-0.15 \mathrm{eV}\right)$, in disagreement with the experiment. On the other hand, the original vdW-DF2 ${ }^{39}$ using rPW86 exchange is too repulsive to cause enough hybridisation and, consequently, it cannot account for the experimental STM images.

Based on physical arguments on the exchange density enhancement factor, we have proposed a combination of vdWDF with PBE exchange to find the balance between dispersion interactions and local hybridisation of the $\mathrm{N}-\mathrm{Cu}$ bonds. This intermediate choice makes satisfactory predictions of the thermodynamical stability of trans, the cis $\rightarrow$ trans barrier, and the main STM features. Furthermore, the use of this functional is also supported by the good agreement with MP2 literature results for the gas-phase H-transfer energetics, and it reproduces the correct $\mathrm{Cu}$ interlayer distance. Thus, we are confident that these results may be generalised to other polycyclic aromatic molecules where intra-molecular H-transfer is feasible and which have similar binding mechanisms on metals, namely where stability of the adsorbate is dictated by the number of undercoodinated atoms that serves as anchoring points to the metal (N, O, S, or even metal atoms) and by the structure and number of aromatic cycles in the molecule.

\section{ACKNOWLEDGMENTS}

We thank T. Kumagai for stimulating discussions. D.N. acknowledges funding from the DIPC. This work has been financially supported by the Basque Departamento de Educación, Universidades e Investigación, the University of the Basque Country UPV/EHU (Grant No. IT-756-13), and the Spanish Ministerio de Economía y Competitividad (Grant No. FIS2013-48286-C2-2-P). J.C.T. acknowledges financial support from the Deutsche Forschungsgemeinschaft (Project No. TR 1109/2-1). Computing resources were provided by the DIPC computing center.

\footnotetext{
${ }^{1}$ A. Lapworth and A. C. Osborn Hann, J. Chem. Soc., Trans. 81, 1508 (1902),

${ }^{2}$ Tautomerism: Methods and Theories, edited by L. Antonov (Wiley-VCH Verlag GmbH \& Co. KGaA, 2013).

${ }^{3}$ P. Liljeroth, J. Repp, and G. Meyer, Science 317, 1203 (2007).

${ }^{4}$ A. Sperl, J. Kröger, and R. Berndt, Angew. Chem., Int. Ed. 50, 5294 (2011).

${ }^{5}$ J. Prasongkit, A. Grigoriev, R. Ahuja, and G. Wendin, Phys. Rev. B 84, 165437 (2011).

${ }^{6}$ W. Auwärter, K. Seufert, D. Ecija, S. Vijayaraghavan, S. Joshi, F. Klappenberger, N. Samudrala, and J. V. Barth, Nat. Nanotechnol. 7, 41 (2012).
} 
${ }^{7}$ G. Bussetti, M. Campione, M. Riva, A. Picone, L. Raimondo, L. Ferraro, C. Hogan, M. Palummo, A. Brambilla, M. Finazzi, L. Duò, A. Sassella, and F. Ciccacci, Adv. Funct. Mater. 24, 958 (2014).

${ }^{8}$ V. D. Pham, V. Repain, C. Chacon, A. Bellec, Y. Girard, S. Rousset, A. Smogunov, Y. J. Dappe, and J. Lagoute, J. Chem. Phys. Lett. 7, 1416 (2016).

${ }^{9}$ T. Kumagai, F. Hanke, S. Gawinkowski, J. Sharp, K. Kotsis, J. Waluk, M. Persson, and L. Grill, Phys. Rev. Lett. 111, 246101 (2013).

${ }^{10}$ T. Kumagai, F. Hanke, S. Gawinkowski, J. Sharp, K. Kotsis, J. Waluk, M. Persson, and L. Grill, Nat. Chem. 6, 41 (2014).

${ }^{11}$ J. N. Ladenthin, L. Grill, S. Gawinkowski, S. Liu, J. Waluk, and T. Kumagai, ACS Nano 9, 7287 (2015).

${ }^{12}$ J. N. Ladenthin, T. Frederiksen, M. Persson, J. C. Sharp, S. Gawinkowski, J. Waluk, and T. Kumagai, Nat. Chem 8, 935 (2016).

${ }^{13}$ G. J. Simpson, S. W. L. Hogan, M. Caffio, C. J. Adams, H. Früchtl, T. van Mourik, and R. Schaub, Nano Lett. 14, 634 (2014).

${ }^{14}$ S. A. Tawfik, X. Y. Cui, S. P. Ringer, and C. Stampfl, J. Chem. Theory Comput. 11, 4154 (2005).

${ }^{15}$ H. H. Kong, Q. Sun, L. K. Wang, Q. G. Tan, C. Zhang, K. Sheng, and W. Xu, ACS Nano 8, 1804 (2014).

${ }^{16}$ C. Zhang, L. Xie, L. K. Wang, H. H. Kong, Q. G. Tan, and W. Xu, J. Am. Chem. Soc. 137, 11795 (2015).

${ }^{17}$ E. Vogel, M. Köcher, H. Schmickler, and J. Lex, Angew. Chem. Int. Ed. 25, 257 (1986).

${ }^{18}$ J. Waluk, "Spectroscopy and tautomerization studies of porphycenes," Chem. Rev. (published online, 2016).

${ }^{19}$ H. Böckmann, S. Liu, J. Mielke, J. Waluk, L. Grill, M. Wolf, and T. Kumagai, Nano Lett. 16, 1034 (2016).

${ }^{20}$ M. F. Shibl, M. Tachikawa, and O. Kühn, Phys. Chem. Chem. Phys. 7, 1368 (2005).

${ }^{21}$ M. F. Shibl, M. Pietrzak, H. H. Limbach, and O. Kühn, ChemPhysChem 8, 315 (2007).

${ }^{22}$ E. C. H. Sykes, L. C. Fernandez-Torres, S. U. Nanayakkara, B. A. Mantooth, R. M. Nevin, and P. S. Weiss, Proc. Natl. Acad. Sci. U. S. A. 102, 17907 (2005).

${ }^{23}$ T. Mitsui, E. Fomin, D. F. Ogletree, M. Salmeron, A. U. Nilekar, and M. Mavrikakis, Angew. Chem., Int. Ed. 46, 5757 (2007).

${ }^{24}$ J. C. Tremblay and M. Blanco-Rey, Phys. Chem. Chem. Phys. 17, 13973 (2015).

${ }^{25}$ J. Klimeš and A. Michaelides, J. Chem. Phys. 137, 120901 (2012).

${ }^{26}$ T. Bucko, J. Hafner, S. Lebegue, and J. G. Angyan, J. Phys. Chem. A 114, 11814 (2010).

${ }^{27}$ P. Santra, J. Klimeš, D. Alfe, A. Tkatchenko, B. Slater, A. Michaelides, R. Car, and M. Scheffler, Phys. Rev. Lett. 107, 185701 (2011).

${ }^{28}$ T. Bjorkman, A. Gulans, A. V. Krasheninnikov, and R. M. Nieminen, Phys. Rev. Lett. 108, 235502 (2012).

${ }^{29}$ J. Carrasco, W. Liu, A. Michaelides, and A. Tkatchenko, J. Chem. Phys. 140, 084704 (2014).

${ }^{30}$ Y. L. Huang, E. Wruss, D. A. Egger, S. Kera, N. Ueno, W. A. Saidi, T. Bucko, A. T. S. Wee, and E. Zojer, Molecules 19, 2969 (2014).

${ }^{31}$ W. Liu, A. Tkatchenko, and M. Scheffler, Acc. Chem. Res. 47, 3369(2014).

${ }^{32}$ R. J. Maurer, V. G. Ruiz, J. Camarillo-Cisneros, W. Liu, N. Ferri, K. Reuter, and A. Tkatchenko, Prog. Surf. Sci. 91, 72 (2016).

${ }^{33}$ L. Martin-Gondre, J. I. Juaristi, M. Blanco-Rey, R. Díez Muiño, and M. Alducin, J. Chem. Phys. 142, 074704 (2015).

${ }^{34}$ M. Wijzenbroek, D. M. Klein, B. Smits, M. Somers, and G. J. Kroes, J. Phys. Chem. A 119, 12146 (2015).

${ }^{35}$ M. Dion, H. Rydberg, E. Schroder, D. C. Langreth, and B. I. Lundqvist, Phys. Rev. Lett. 92, 246401 (2004).

${ }^{36}$ T. Thonhauser, V. R. Cooper, S. Li, A. Puzder, P. Hyldgaard, and D. C. Langreth, Phys. Rev. B 76, 125112 (2007).

${ }^{37}$ A. Gulans, M. J. Puska, and R. M. Nieminen, Phys. Rev. B 79, 201105(R) (2009).
${ }^{38}$ G. Kresse and J. Furthmüller, Phys. Rev. B 54, 11169 (1996).

${ }^{39}$ K. Lee, E. D. Murray, L. Kong, B. I. Lundqvist, and D. C. Langreth, Phys. Rev. B 82, 081101 (2010).

${ }^{40}$ J. Klimeš, D. R. Bowler, and A. Michaelides, Phys. Rev. B 83, 195131 (2011).

${ }^{41}$ J. Klimeš, D. R. Bowler, and A. Michaelides, J. Phys.: Condens. Matter 22, 022201 (2009).

${ }^{42}$ G. Román-Pérez and J. M. Soler, Phys. Rev. Lett. 103, 096102 (2009).

${ }^{43}$ P. Jurěcka, J. Šponer, J. Černy, and P. Hobza, Phys. Chem. Chem. Phys. 8, 1985 (2006).

${ }^{44}$ K. Berland and P. Hyldgaard, Phys. Rev. B 89, 035412 (2014).

${ }^{45}$ I. Hamada, Phys. Rev. B 89, 121103(R) (2014).

${ }^{46}$ K. Berland, V. R. Cooper, K. Lee, E. Schröder, T. Thonhauser, P. Hyldgaard, and B. I. Lundqvist, Rep. Prog. Phys. 78, 066501 (2015).

${ }^{47}$ S. Grimme, J. Antony, S. Ehrlich, and S. Krieg, J. Chem. Phys. 132, 154104 (2010).

${ }^{48}$ A. Tkatchenko and M. Scheffler, Phys. Rev. Lett. 102, 073005 (2009).

${ }^{49}$ V. G. Ruiz, W. Liu, E. Zojer, M. Scheffler, and A. Tkatchenko, Phys. Rev. Lett. 108, 146103 (2012).

${ }^{50}$ A. Tkatchenko, R. A. DiStasio, R. Car, and M. Scheffler, Phys. Rev. Lett. 108, 236402 (2012).

${ }^{51}$ A. Ambrosetti, A. M. Reilly, R. A. DiStasio, Jr., and A. Tkatchenko, J. Chem. Phys. 140, 18A508 (2014).

${ }^{52}$ R. J. Maurer, V. G. Ruiz, and A. Tkatchenko, J. Chem. Phys. 143, 102808 (2015).

${ }^{53}$ P. E. Blöchl, Phys. Rev. B 50, 17953 (1994).

${ }^{54}$ H. J. Monkhorst and J. D. Pack, Phys. Rev. B 13, 5188 (1976).

${ }^{55}$ S. R. Bahn and K. W. Jacobsen, Comput. Sci. Eng. 4, 56 (2002).

${ }^{56}$ E. Bitzek, P. Koskinen, F. Gähler, M. Moseler, and P. Gumbsch, Phys. Rev. Lett. 97, 170201 (2006).

${ }^{57}$ C. Lee, W. Yang, and R. G. Parr, Phys. Rev. B 37, 785 (1988).

${ }^{58}$ A. D. Becke, J. Chem. Phys. 98, 5648 (1993).

${ }^{59}$ C. Møller and M. S. Plesset, Phys. Rev. B 46, 618 (1934).

${ }^{60}$ M. Head-Gordon, J. A. Pople, and M. J. Frisch, Chem. Phys. Lett. 153, 503 (1988)

${ }^{61}$ P. M. Kozlowski, M. Z. Zgierski, and J. Baker, J. Chem. Phys. 109, 5905 (1998)

${ }^{62}$ Z. Smedarchina, M. F. Shibl, O. Kühn, and A. Fernández-Ramos, Chem. Phys. Lett. 436, 314 (2007).

${ }^{63}$ J. Waluk, M. Müller, P. Swiderek, M. Köcher, E. Vogel, G. Hohlneicher, and J. Michl, J. Am. Chem. Soc. 113, 5511 (1991).

${ }^{64}$ W. Liu, F. Maass, M. Willenbockel, C. Bronner, M. Schulze, S. Soubatch, F. S. Tautz, P. Tegeder, and A. Tkatchenko, Phys. Rev. Lett. 115, 036104 (2015).

${ }^{65}$ Atoms in Molecules: A Quantum Theory, edited by R. F. W. Bader (Oxford University Press, Oxford, UK, 1990).

${ }^{66}$ J. P. Perdew and Y. Wang, Phys. Rev. B 33, 8800(R) (1986).

${ }^{67}$ E. D. Murray, K. Lee, and D. C. Langreth, J. Chem. Theory Comput. 5, 2754 (2009).

${ }^{68}$ A. D. Becke, J. Chem. Phys. 85, 7184 (1986).

${ }^{69}$ A. D. Becke, Phys. Rev. A 38, 3098 (1988).

${ }^{70}$ A. D. Becke, J. Chem. Phys. 84, 4524 (1986).

${ }^{71}$ B. Hammer, L. B. Hansen, and J. K. Nørskov, Phys. Rev. B 59, 7413 (1999).

${ }^{72}$ E. H. Lieb and S. Oxford, Int. J. Quantum Chem. 19, 427 (1981).

${ }^{73}$ F. Bischoff, K. Seufert, W. Auwärter, S. Joshi, S. Vijayaraghavan, D. Ecija, K. Diller, A. C. Papageorgiou, S. Fischer, F. Allegretti, D. A. Duncan, F. Klappenberger, F. Blobner, R. Y. Han, and J. V. Barth, ACS Nano 7, 3139 (2013).

${ }^{74}$ M. Müller, K. Diller, R. Maurer, and K. Reuter, J. Chem. Phys. 144, 024701 (2016).

${ }^{75}$ H. Piwoński, C. Stupperich, A. Hartschuh, J. Sepioł, A. Meixner, and J. Waluk, J. Am. Chem. Soc. 127, 5302 (2005). 